\title{
Penile squamous cell carcinoma: a review of the literature and case report treated with Mohs micrographic surgery *
}

\author{
Elizabeth Marchionne ${ }^{1}$ \\ Andrea Hui ${ }^{2}$
}

\author{
Caroline Perez ${ }^{1}$ \\ Amor Khachemoune ${ }^{2}$
}

DOI: http://dx.doi.org/10.1590/abd1806-4841.20175009

\begin{abstract}
The majority of penile carcinoma is squamous cell carcinoma. Although uncommon in the United States, it represents a larger proportion of cancers in the underdeveloped world. Invasive squamous cell carcinoma may arise from precursor lesions or de novo, and has been associated with lack of circumcision and HPV infection. Early diagnosis is imperative as lymphatic spread is associated with a poor prognosis. Radical surgical treatment is no longer the mainstay, and penile sparing treatments now are often used, including Mohs micrographic surgery. Therapeutic decisions should be made with regard to the size and location of the tumor, as well as the functional desires of the patient. It is critical for the dermatologist to be familiar with the evaluation, grading/staging, and treatment advances of penile squamous cell carcinoma. Herein, we present a review of the literature regarding penile squamous cell carcinoma, as well as a case report of invasive squamous cell carcinoma treated with Mohs micrographic surgery.
\end{abstract}

Keywords: Carcinoma, squamous cell; Mohs surgery; Penile neoplasms; Penis

\section{INTRODUCTION}

Penile cancer is a rare diagnosis in the United States and other developed nations. In the United States in 2015, the American Cancer Society predicted 1,820 new cases with 310 penile cancer deaths. ${ }^{1}$ However, the disease is more common in developing countries, where it may represent up to $10 \%$ of cancers in males. ${ }^{2,3,4}$ The majority of penile cancer is squamous cell carcinoma (SCC). ${ }^{5}$

The pathogenesis of penile cancer is not entirely known. SCC may evolve from penile intraepithelial neoplasia (PIN) or arise de novo. ${ }^{4}$ Penile SCC has been associated with high-risk HPV infections, most commonly strains 16 and 18. The mechanism through which HPV leads to penile cancer is most likely mediated through viral oncogenes E6 and E7, which are actively transcribed by HPV-infected cells. Viral oncogene E6 targets p53 and viral oncogene E7 targets RB1; both p53 and RB1 are tumor suppressor genes, which are negative regulators of cell proliferation. When these genes are transformed, this may lead to unchecked proliferation and subsequent development of malignancy. ${ }^{3}$ HPV-DNA has been found in only $22-72 \%$ of penile SCC, but has been identified in the vast majority of PIN (70-100\%). ${ }^{3}$ This discordance between the presence of HPV-DNA in SCC versus PIN suggests an HPV independent and dependent etiology for the development of SCC. ${ }^{4}$

The prevalence of genital HPV-DNA in men is $1.3 \%$ to $72.9 \%$, with a mean of $>20 \%$ reported by most studies. ${ }^{6}$ In several cohort studies, it is estimated that most HPV infections in men clear in less than 12 months, with a median of 5.9 to 7.5 months. ${ }^{7,8}$ Increasing age and fewer numbers of female partners are positively correlated with a greater probability of clearing an HPV infection. ${ }^{8}$ The development of the quadrivalent HPV vaccine to high-risk strains 6, 11,16 , and 18 , has been shown to significantly reduce HPV-associated genital disease in men, including genital warts, with an efficacy of $89.4 \% .^{9}$ The use of the HPV vaccine in males has the potential to significantly reduce the incidence of penile cancer, among other disfiguring genital lesions such as condylomas and genital warts.

Penile SCC occurs almost exclusively in uncircumcised men. ${ }^{3}$ Neonatal circumcision is implicated as strongly protective

Received on 04.08.2015

Approved by the Advisory Board and accepted for publication on 28.10.2015

* Study conducted at the Downstate and Veterans Affairs Medical Center - Brooklyn (NY), United States.

Financial Support: None.

Conflict of Interest: None.

School of medicine - University of Nevada - Reno, United States.

Department of Dermatology - State University of New York - Downstate and Veterans Affairs Medical Center - Brooklyn (NY), United States.

C 2017 by Anais Brasileiros de Dermatologia

An Bras Dermatol. 2017;92(1):95-9. 
against penile cancer. ${ }^{10,11}$ Proposed etiological factors associated with an intact foreskin are smegma and phimosis. Smegma is a product of desquamating epithelial cells and bacterial byproducts found between the glans penis and foreskin. ${ }^{11}$ Smegma-induced chronic inflammation may predispose to the development of phimosis as well as SCC. ${ }^{4}$ Phimosis, the inability to retract the foreskin over the glans, has been found in $25-60 \%$ of patients with penile cancer. ${ }^{3,4}$ It is associated with an increased incidence of infection as well as dysplastic changes of the preputial sac. ${ }^{12}$ Further, uncircumcised men have been found to be at an increased risk of HPV infection when compared to their circumcised counterparts. ${ }^{13}$ Additional risk factors associated with the development of penile SCC include smoking, HIV infection, poor genital hygiene, previous injury to the penis, chronic balanitis, lichen sclerosis et atrophicus, and psoralen plus ultraviolet A treatment to the area. ${ }^{3,4,14}$

Penile SCC most commonly presents between the ages of 50 and 70 years. ${ }^{3}$ The majority of lesions are found on the glans (48\%), followed by the prepuce $(21 \%)$, both glans and prepuce $(15 \%)$, coronal sulcus $(6 \%)$, and shaft $(<2 \%) .{ }^{3}$ Clinical presentation is variable. It may present as a small area of induration and erythema or a large ulcerating and infiltrative lesion. As the disease progresses, there may be associated itching, bleeding, discharge, foul odor, and pain. ${ }^{3}$ Presentation may be delayed secondary to psychological factors, with an estimated 15 to $60 \%$ of patients postponing presentation for at least one year. ${ }^{3,4}$ Despite this, most men $(66 \%)$ initially present with localized disease. ${ }^{4}$ Assessment of lymphatic spread with palpation of inguinal lymph nodes is an essential component of the initial physical exam. Lymphatic spread usually occurs in a predictable course, first to the superficial and deep inguinal nodes, followed by the pelvic, and then periaortic nodes. ${ }^{15}$ Distant metastases are generally uncommon (1-10\%) and occur late in the disease. ${ }^{3,15}$

Differential diagnostic considerations of penile SCC include premalignant and malignant lesions, infections, and inflammatory conditions. Neoplastic lesions such as erythroplasia of Queyrat, Bowen's disease or Bowenoid papulosis should be considered. ${ }^{4}$ Condyloma acuminata may resemble SCC, especially the verrucous variant. Ulceration and lymphadenopathy may raise concern for the chancre of a primary syphilis infection or the chancroid of Haemophilus ducreyi. Additionally, the psoriatic scales and plaques of genital psoriasis and violaceous lesions of lichen planus may appear clinically similar to SCC. ${ }^{14}$

Penile SCC can be divided into several subtypes. The most common subtypes include usual SCC (48-65\%), basaloid carcinoma (4-10\%), warty carcinoma (7-10\%), verrucous carcinoma (3-8\%), papillary carcinoma (5-15\%), and mixed carcinomas (9-10\%). ${ }^{16}$ Each subtype has distinct histologic features. Histopathologic analysis is used to grade the tumor, which is then assigned on a spectrum based on cellular differentiation (Chart 1) ${ }^{14,16,17}$ Staging is performed using the TNM penile cancer system developed by the American Joint Committee on Cancer (Chart 2). ${ }^{17}$ Staging is based on depth of tumor invasion, nodal involvement, and distant metastases (Chart 3)..$^{17}$

Surgical decisions should be made with regard to the size and location of the tumor, as well as the functional desires of the patient. For patients with PIN, topical imiquimod or 5-fluoroura- cil, circumcision and local excision, and laser ablative therapy could be used as penile preserving techniques. ${ }^{14}$ When treating patients with well- to moderately-differentiated T1 SCC tumors, penile sparing surgery should be utilized. ${ }^{14}$ In these stage I tumors, wide local

CHART 1: Histopathological grading of penile SCC

\begin{tabular}{ll} 
Pathological Grading (G) \\
\hline GX & Grade cannot be assessed \\
G1 & Well differentiated \\
G2 & Moderately differentiated \\
G3 & Poorly differentiated \\
G4 & Undifferentiated
\end{tabular}

Reproduced with the permission of the American Joint Committee on Cancer (AJCC), Chicago, Illinois. The original source for this material is the AJCC Cancer Staging Manual, Seventh Edition (2010) published by Springer Science and Business Media LLC, www.springer.com.

Chart 2: American Joint Committee on Cancer TMN classification for penile cancer

Primary Tumor (T)

TX Primary tumor cannot be assessed

T0 No evidence of primary tumor

Tis Carcinoma in situ

Ta Noninvasive verrucous carcinoma*

T1a Tumor invades subepithelial connective tissue without lymph vascular invasion and is not poorly differentiated (i.e., grade 3-4)

T1b Tumor invades subepithelial connective tissue with lymph vascular invasion and is poorly differentiated

T2 Tumor invades corpus spongiosum or cavernosum

T3 Tumor invades urethra

T4 Tumor invades other adjacent structures

* Note: Broad pushing penetration (invasion) is permitted; destructive invasion is against this diagnosis.

\section{Regional Lymph Nodes (N)}

\section{Clinical Stage Definition*}

cNX Regional lymph nodes cannot be assessed

cN0 No palpable or visibly enlarged inguinal lymph nodes

cN1 Palpable mobile unilateral inguinal lymph node

cN2 Palpable mobile multiple or bilateral inguinal lymph nodes

cN3 Palpable fixed inguinal lymph nodal mass or pelvic lymphadenopathy unilateral or bilateral

* Note: Clinical stage definition based on palpation, imaging

\section{Pathologic Stage Definition*}

pNX Regional lymph nodes cannot be assessed

pN0 No regional lymph node metastasis

pN1 Metastasis in a single inguinal lymph node

pN2 Metastasis in multiple or bilateral inguinal lymph nodes

pN3 Extranodal extension of lymph node metastasis or pelvic lymph node(s) unilateral or bilateral

*Note: Pathologic stage definition based on biopsy or surgical excision.

\section{Distant Metastasis (M)}

M0 No distant metastasis

M1 Distant metastasis*

*Note: Lymph node metastasis outside of the true pelvis in addition to visceral or bone sites.

Reproduced with the permission of the American Joint Committee on Cancer (AJCC), Chicago, Illinois. The original source for this material is the AJCC Cancer Staging Manual, Seventh Edition (2010) published by Springer Science and Business Media LLC, www.springer.com. 
CHART 3: American Joint Committee on Cancer penile cancer staging

\begin{tabular}{|llll|}
\hline \multicolumn{2}{|l}{ Anatomic Stage/Prognostic Groups } & \\
\hline Stage 0 & Tis & N0 & M0 \\
& Ta & N0 & M0 \\
Stage I & T1a & N0 & M0 \\
Stage II & T1b & N0 & M0 \\
& T2 & N0 & M0 \\
& T3 & N0 & M0 \\
Stage IIIa & T1-3 & N1 & M0 \\
Stage IIIb & T1-3 & N2 & M0 \\
Stage IV & T4 & Any N & M0 \\
& Any T & N3 & M0 \\
& Any T & Any N & M1 \\
\hline
\end{tabular}

Reproduced with the permission of the American Joint Committee on Cancer (AJCC), Chicago, Illinois. The original source for this material is the AJCC Cancer Staging Manual, Seventh Edition (2010) published by Springer Science and Business Media LLC, www.springer.com.

excision using $2 \mathrm{~cm}$ margins has been traditionally used; however, smaller 5 to $10 \mathrm{~mm}$ margins have shown low recurrence rates. ${ }^{14}$ Mohs micrographic surgery (MMS) is becoming increasingly popular and has the ability to provide superior aesthetic results with preservation of normal surrounding tissue..$^{15,18,19}$

A great advantage of using MMS is removal of the tumor with complete visualization to ensure negative margins. ${ }^{18,19}$ For penile SCC, MMS is most successful in smaller tumors with lower grade. ${ }^{18}$ If urethral involvement is present, MMS should be discouraged. ${ }^{19}$ Patients with tumors staged II and above are usually not candidates for penile sparing therapy due to larger tumor size and further depth of invasion. ${ }^{14,15}$ Partial or total penectomy should be considered in these patients. Concern should be taken for preservation of urination and sexual function. ${ }^{20}$ Radiation and chemotherapy could be used as adjuvant therapy or in cases of palliation. ${ }^{15}$

Evaluation of lymphatic spread is critical and guidelines are still emerging. ${ }^{5}$ About $28-64 \%$ of patients will present with palpable inguinal nodes.$^{21}$ However, of these, $47-85 \%$ will have metastatic disease, with the remainder of nodal enlargement secondary to inflammation. ${ }^{4,21}$ Even in patients without palpable nodes, an estimated $25 \%$ will still have micrometastatic disease. ${ }^{14}$ Inguinal lymph node dissection is a diagnostic and potentially curative procedure. ${ }^{14,22}$ However, due to potential complications, it is preferred to only perform this procedure in patients at a high risk for metastasis. ${ }^{15,22}$ Techniques such as MRI, PET scan, fine needle aspiration, and dynamic sentinel node biopsy may precede an inguinal lymph node dissection. ${ }^{15}$ Minimally invasive techniques such as laparoscopic or robotic-assisted inguinal lymphadenectomy are evolving. ${ }^{22,23}$ A multidisciplinary treatment team involving medical oncology, radiation oncology, urology, and psychiatry in addition to dermatology may be warranted. ${ }^{15,24}$

Prognosis is good for localized disease. The most important prognostic factor is the extent of nodal metastasis. ${ }^{15,21}$ The 5-year cancer specific survival of a primary SCC with no inguinal metastasis is $85-100 \%$, one positive node is 79 to $89 \%$, bilateral or multiple nodal metastasis is 17 to $60 \%$, and metastasis to pelvic nodes is 0 to $17 \%{ }^{21}$ Prognosis with extranodal metastasis is poor. ${ }^{21}$

\section{CASE REPORT}

A 60-year-old men presented for evaluation of a penile lesion present for one year. The patient denied any associated pain, bleeding, odor, discharge, or dysuria. There were no recent changes to the lesion. The patient denied history of sexually transmitted infections and reported being in a monogamous relationship with his girlfriend for 25 years. He denied any erectile dysfunction. The patient was otherwise healthy and family history was noncontributory. Physical exam was significant for a $1.5 \times 1.5 \mathrm{~cm}$ erythematous ulcerated plaque on the inner foreskin $3 \mathrm{~cm}$ from the urethral tip and $1 \mathrm{~cm}$ from the coronal sulcus (Figure 1). Inguinal lymphadenopathy was not appreciated on palpation, however exam was limited due to the presence of bilateral inguinal hernias.

A shave biopsy was obtained and revealed invasive squamous cell carcinoma, moderately differentiated (Figure 2). The decision was made to proceed with Mohs micrographic surgery (MMS) and to refer the patient to urology for evaluation of inguinal nodes following MMS. Imaging to determine if enlarged nodes were present was not performed preoperatively.

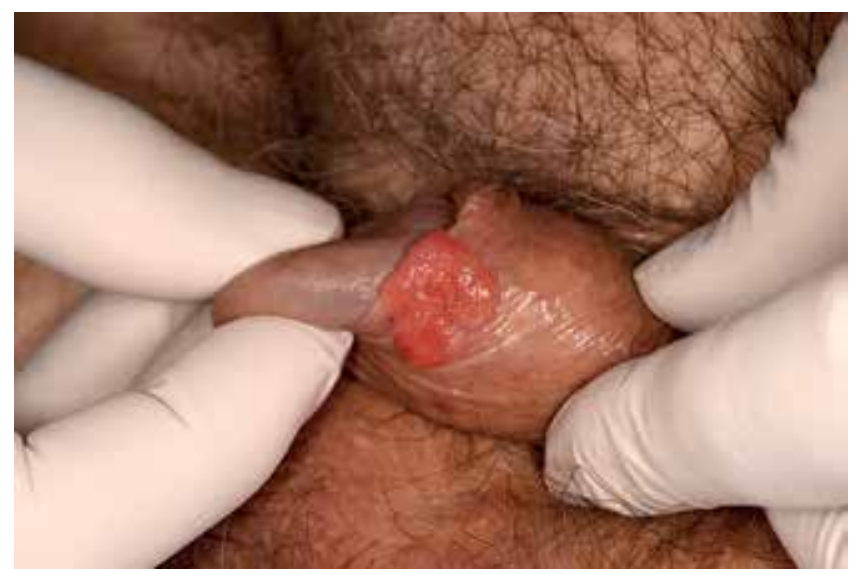

FIGURE 1: $1.5 \mathrm{~cm}$ x $1.5 \mathrm{~cm}$ ulcerated and erythematous lesion on the penis

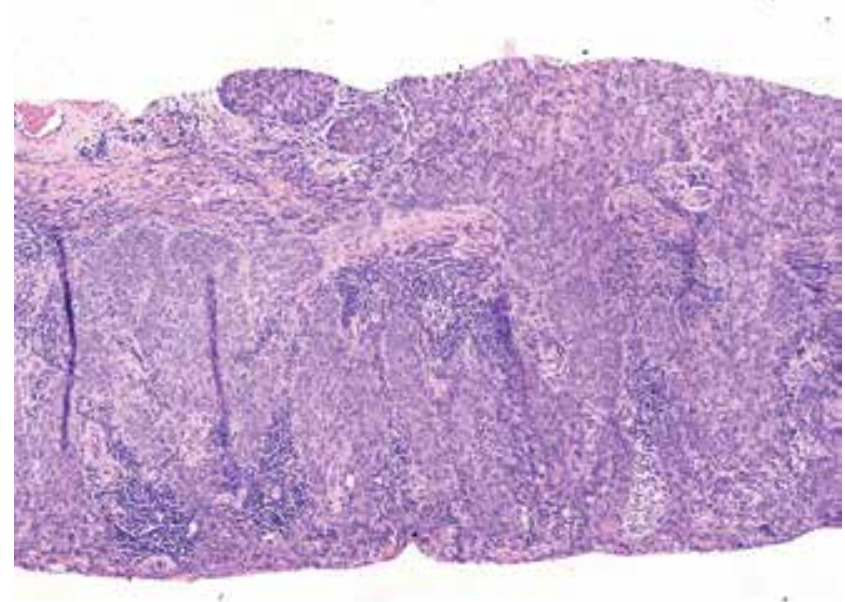

FIGURE 2: Squamous cell carcinoma, moderately differentiated. Atypical cells extend into the deep dermis. (Hematoxylin \& eosin $\mathrm{x} 10)$ 


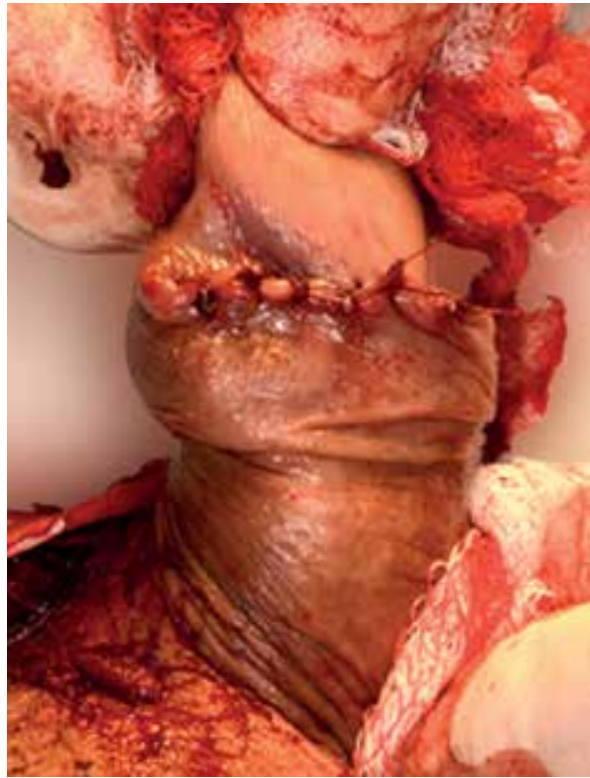

Figure 3:

Linear closure of the defect, immediately post-operative
The tumor bulk was excised, followed by one stage of MMS with excision of the borders as a horizontal layer, 2-3 mm in thickness. The tissue was mapped, frozen, cut, and stained with toluidine blue. Microscopic evaluation confirmed the tumor was completely excised with no further disease in the deep outer borders. The lesion invaded into the deep dermis with no invasion into adjacent structures. The defect was repaired in the office using linear closure (Figure 3).

The consultant urologist concluded that the patient's inguinal hernias limited nodal evaluation and recommended hernia repair prior to further assessment. The patient was seen by general surgery for evaluation, however he refused further interventions.

The patient was followed for over one year with no evidence of recurrence of the lesion and no functional concerns.

Squamous cell carcinoma of the penis is a rare diagnosis. Therapeutic decisions should be made with regard to the size and location of the tumor, as well as with consideration to the functional desires of the patient. Mohs micrographic surgery is an excellent alternative for patients with tumors of low grade and stage.]

\section{REFERENCES}

1. American Cancer Society. Cancer Facts and Figures 2015. Atlanta, Ga: American Cancer Society; 2015. [cited 2015 May 12]. Available from: http://www.cancer. org/cancer/penilecancer/detailedguide/penile-cancer-key-statistics

2. Pow-Sang MR, Ferreira U, Pow-Sang JM, Nardi AC, Destefano V. Epidemiology and natural history of penile cancer. Urology. 2010;76:S2-6.

3. Barnholtz-sloan JS, Maldonado JL, Pow-sang J, Giuliano AR, Guiliano AR. Incidence trends in primary malignant penile cancer. Urol Oncol. 2007;25:361-7.

4. Brady KL, Mercurio MG, Brown MD. Malignant tumors of the penis. Dermatol Surg. 2013;39:527-47.

5. Burt LM, Shrieve DC, Tward JD. Stage presentation, care patterns, and treatment outcomes for squamous cell carcinoma of the penis. Int J Radiat Oncol Biol Phys. 2014;88:94-100.

6. Dunne EF, Nielson CM, Stone KM, Markowitz LE, Giuliano AR. Prevalence of HPV infection among men: a systematic review of the literature. J Infect Dis. 2006;194:1044-57.

7. Lu B, Wu Y, Nielson CM, Flores R, Abrahamsen M, Papenfuss M, et al. Factors associated with acquisition and clearance of human papillomavirus infection in a cohort of US men: a prospective study. J Infect Dis. 2009;199:362-71.

8. Giuliano AR, Lee JH, Fulp W, Villa LL, Lazcano E, Papenfuss MR, et al. Incidence and clearance of genital human papillomavirus infection in men (HIM): a cohort study. Lancet. 2011;377:932-40.

9. Giuliano AR, Palefsky JM, Goldstone S, Moreira ED Jr, Penny ME, Aranda C, et al Efficacy of quadrivalent HPV vaccine against HPV Infection and disease in males. N Engl J Med. 2011;364:401-11.
10. Larke NL, Thomas SL, dos Santos Silva I, Weiss HA. Male circumcision and penile cancer: a systematic review and meta-analysis. Cancer Causes Control. 2011;22:1097-110.

11. Morris BJ, Gray RH, Castellsague X, Bosch FX, Halperin DT, Waskett JH, et al. The Strong Protective Effect of Circumcision against Cancer of the Penis. Adv Urol. 2011;2011:812368.

12. Reddy CR, Devendranath V, Pratap S. Carcinoma of penis--role of phimosis. Urology. 1984;24:85-8.

13. Castellsagué $X$, Bosch FX, Muñoz N, Meijer CJ, Shah KV, de Sanjose S, et al. Male circumcision, penile human papillomavirus infection, and cervical cancer in female partners. N Engl J Med. 2002;346:1105-12.

14. Spiess PE, Horenblas S, Pagliaro LC, Biagioli MC, Crook J, Clark PE, et al. Current concepts in penile cancer. J Natl Compr Canc Netw. 2013;11:617-24

15. McDougal WS, Lee RJ, Efstathiou JA, Harisinghani M, Wu CL. Case records of the Massachusetts General Hospital. Case 2-2014. A 44-year-old man with a lesion on the penis. N Engl J Med. 2014;370:263-71.

16. Chaux A, Cubilla AL. Advances in the pathology of penile carcinomas. Hum Pathol. 2012;43:771-89.

17. Edge SB, Byrd DR, Compton CC, Fritz AG, Greene FL, Trotti A, editors. AJCC Cancer Staging Manual. 7th ed. New York, NY: Springer; 2010.

18. Shindel AW, Mann MW, Lev RY, Sengelmann R, Petersen J, Hruza GJ, et al. Mohs micrographic surgery for penile cancer: management and long-term followup. J Urol. 2007;178:1980-5. 
19. Wells MJ, Taylor RS. Mohs micrographic surgery for penoscrotal malignancy. Urol Clin North Am. 2010;37:403-9.

20. Kieffer JM, Djajadiningrat RS, van Muilekom EA, Graafland NM, Horenblas S, Aaronson NK. Quality of life in patients treated for penile cancer. J Urol. 2014;192:1105-10.

21. Ficarra V, Akduman B, Bouchot 0, Palou J, Tobias-Machado M. Prognostic factors in penile cancer. Urology. 2010;76:S66-73.

22. Kharadjian TB, Matin SF, Pettaway CA. Early experience of robotic-assisted inguinal lymphadenectomy: review of surgical outcomes relative to alternative approaches. Curr Urol Rep. 2014;15:412.

23. Matin SF, Cormier JN, Ward JF, Pisters LL, Wood CG, Dinney CP, et al. Phase 1 prospective evaluation of the oncological adequacy of robotic assisted videoendoscopic inguinal lymphadenectomy in patients with penile carcinoma. BJU Int. 2013;111:1068-74.

24. Yeung LL, Brandes SB. Dynamic sentinel lymph node biopsy as the new paradigm for the management of penile cancer. Urol Oncol. 2013:31:693-6
MAILING ADDRESS:

Amor Khachemoune

Veterans Affairs Medical Center

800 Poly Place

Brooklyn, NY 11209.

Email: Amorkh@gmail.com

How to cite this article: Marchionne E, Perez C, Hui A, Khachemoune A. Penile squamous cell carcinoma: a review of the literature and case report treated with Mohs micrographic surgery . An Bras Dermatol. 2017;92(1):95-9. 